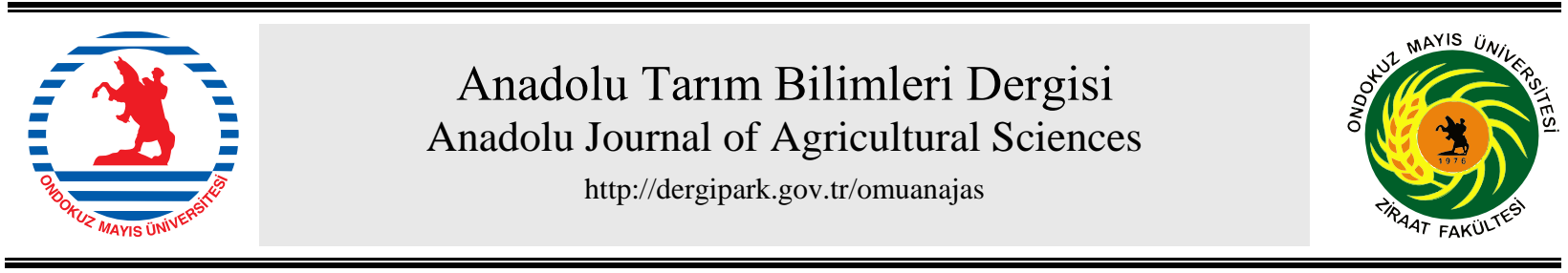

\title{
The Effect of Boron Stress on Germination Properties of Pepper, Eggplant and Watermelon Seeds Subjected to Salicylic Acid Pre-application
}

\author{
Ahmet Turhan ${ }^{\mathrm{a}, *}$, (1) Hayrettin Kuşçu ${ }^{\mathrm{b}}$ \\ ${ }^{a}$ Bursa University of Uludag, Mustafakemalpasa Vocational School, Department of Plant and Animal Production, Bursa, Turkey. \\ ${ }^{b}$ University of Uludag, Faculty of Agriculture, Department of Biosystems Engineering, Bursa, Turkey; \\ *Sorumlu yazar/corresponding author: turhan@uludag.edu.tr \\ Geliş/Received 13/11/2020～Kabul/Accepted 24/05/2021
}

\begin{abstract}
Boron toxicity constitutes a limiting factor on seed germination. Salicylic acid-like plant growth regulators play an important role in alleviation the negative effects of boron stress. The research was carried out to determine the germination properties of seeds (pepper, eggplant, watermelon) with and without salicylic acid (SA) pre-application at different boron (B) concentrations. Experiments were conducted in randomized plots design with 4 replications. Seed germination percentage, mean germination time, germination index and germination energy values were determined under four different SA $(0,0.5,1.0$ and $2.0 \mathrm{mM})$ and six different $\mathrm{B}\left(0,1.0,2.0,4.0,8.0\right.$ and $\left.16.0 \mathrm{mg} \mathrm{L}^{-1}\right)$ doses. The greatest germination percentage, index and energy values and the shortest mean germination time were obtained from $1.0 \mathrm{mg} \mathrm{L}^{-1} \mathrm{~B}$ dose, on the other hand, increasing $\mathrm{B}$ stress negatively influenced germination parameters. Especially at $4.0,8.0$ and $16.0 \mathrm{mg} \mathrm{L}^{-1} \mathrm{~B}$ stress, $1.0 \mathrm{mM} \mathrm{SA}$ supplemented into growing media increased germination parameters of all three vegetable species, reduced the negative effects of boron and positively influenced germination time. Present findings revealed that SA could be used as an efficient tool in alleviation of negative effects of boron toxicity. On the other hand, SA applications were not effective or had quite limited effects on non-B application or low B-application $\left(<1.0 \mathrm{mg} \mathrm{L}^{-1}\right)$ seeds.
\end{abstract}

\section{Salisilik Asit Ön Uygulamasına Tabi Tutulmuş Biber, Patlıcan ve Karpuz Tohumlarında Bor Stresinin Çimlenme Özellikleri Üzerine Etkisi}

\section{ÖZET}

Tarımsal üretimde tohumun çimlenme özelliklerini sınırlandıran önemli faktörlerden birisi de bor toksisitesidir. Salisilik asit gibi bitki büyüme düzenleyicileri, bor stresinin olumsuz etkisini hafifletmede önem taşımaktadır. Araştırma, salisilik asit (SA) ön uygulaması yapılmış ve yapılmamış tohumların (biber, patlıcan, karpuz) farklı bor (B) konsantrasyonlarındaki çimlenme özelliklerini belirlemek amacıyla yapılmıştır. Laboratuvar şartlarında yapılan bu deneme, tesadüf parselleri deneme desenine uygun, dört SA $(0,0.5,1.0$ ve $2.0 \mathrm{mM})$ ve altı $\mathrm{B}$ dozu $\left(0,1.0,2.0,4.0,8.0\right.$ ve $\left.16.0 \mathrm{mg} \mathrm{L}^{-1}\right)$ kullanılarak 4 tekerrürlü olarak kurulmuştur. Çalışmada tohumların çimlenme yüzdesi, ortalama çimlenme süresi, çimlenme indeksi ve enerjisindeki değişimler incelenmiştir. En yüksek çimlenme özelliklerini (çimlenme, yüzdesi, indeksi ve enerjisi) ve en kısa çimlenme süresini $1.0 \mathrm{mg} \mathrm{L}^{-1}$ B dozu göstermiş, buna karşın artan B stresi bu değerleri olumsuz etkilemiştir. Özellikle, 4.0,
Keywords:

Boron toxicity

Germination

percentage

Germination index

Germination energy
Anahtar Sözcükler:

Bor toksisitesi

Çimlenme yüzdesi

Çimlenme indeksi

Çimlenme enerjisi

(C) OMU ANAJAS 2021 
8.0 ve $16.0 \mathrm{mg} \mathrm{L}^{-1} \mathrm{~B}$ stresinde, ortama ilave edilen $1.0 \mathrm{mM}$ SA her üç sebzede de çimlenme özelliklerini artırarak, borun zararlı etkilerini azaltabilmiş ve çimlenme süresini de olumlu etkilemiştir. Bu durum, toksisite riski ile ortaya çıkan olumsuzlukların giderilmesinde SA'in kullanılabilecek bir alternatif olabileceğini gösterilmiş̧ir. Buna karşın, B uygulanmayan veya düşük seviyede $\mathrm{B}\left(<1.0 \mathrm{mg} \mathrm{L}^{-1}\right)$ uygulanan tohumlarda, SA uygulamalarının etkisi olmamış veya oldukça sınırlı kalmıştır.

\section{Introduction}

Boron is an essential micronutrient for normal growth and development of plants (Brdar-Jokanoviç, 2020). Among the plant micronutrients such as $\mathrm{Fe}, \mathrm{Zn}, \mathrm{Cu}, \mathrm{Mn}$ and etc. boron is the only non-metallic element and it exists in borate forms in the nature. Among these forms, borax is the most abundant one (Adriano, 1986). Boron is basically up taken by the plants as dissociated boric acid through passive absorption and also actively absorbed as borate ions.

Germination is an important agronomic trait deeply influencing plant growth and productivity. Alamri et al. (2018) indicated effects of soil boron concentrations on germination and seedling growth parameters and reported that low boron concentrations $\left(0.5 \mathrm{mg} \mathrm{L}^{-1}\right)$ had positive effects on germination and seedling growth parameters, but high concentrations $\left(1.0 \mathrm{mg} \mathrm{L}^{-1}\right)$ reduced germination rates and seedling growth parameters significantly. In pepper plants, boron toxicity symptoms (slight chlorosis along the leaf edges) were encountered when the boron concentration of nutrient solution increased to $5.0 \mathrm{mg} \mathrm{L}^{-1}$ (Sarafi et al., 2017). Decreasing germiantion ratios and germination index values of pepper plants were reported at boron concentrations of over $2.0 \mathrm{mg} \mathrm{L}^{-1}$ (Turhan, 2018).

Salicylic acid is defined as an internal plant growth regulator effecting plant growth and development under stress conditions (Hayat et al., 2010). Besides salinity and drought stress, salicylic acid is also used to provide resistance against adverse conditions like high and low temperature stress, heavy metal and frost stress (Baktır, 2010; Kumlay and Eryiğit, 2011). Previous studies revealed that external plant growth regulator treatments such as salicylic acid (SA), abscisic acid (ABA), gibberellic acid ( $\mathrm{GA}_{3}$ ) and jasmonic acid (JA) positively influenced plant growth and development under stress conditions (Datta et al., 1997; Kaydan and Yağmur, 2006; Ünver and Tilki, 2012; Y1ld1z et al., 2014). Besides, it was reported that SA retarded seed germination of Arabidopsis under normal conditions, on the other hand, reduced oxidative stress under high salt concentrations and thus stimulated seed germination (Lee et al., 2010). It was also reported that SA influenced seed germination, but such effects varied with the concentrations (Rajjou et al., 2006; Singh et al., 2010). Baran et al. (2019) reported that negative effects of salt stress could be alleviated with SA treatments. Boukraâ et al. (2015) reported based on germination rate and growth parameters that inhibitor effect of high salinity in chickpea was significantly reduced by SA treatments. In beans, low dose SA treatments $(0.25 \mathrm{mM})$ increased germination ratios (Dadaşoğlu and Ekinci, 2013).

Number of studies about the effects of SA applications on growth and development of plants under B stress is insufficient and there is much to investigate in this subject matter. Therefore, this study was conducted to investigate the effects of SA applications some germination parameters of three different vegetables species (pepper, eggplant and watermelon) under the B stress.

\section{Materials and Methods}

\subsection{Material and experimental site}

Experiments were conducted in seed laboratories of Mustafakemalpasa Vocational School of Bursa Uludag University in the year 2020. Industrial pepper (Capsicum annuum L., cv. Kapija), eggplant (Solanum melongena L., $c v$. Elmur) and watermelon (Citrullus lanatus $c v$. Crimson Sweet) species were used as the plant material of the study.

\subsection{Salicylic acid treatments}

Sufficient number of sturdy seeds with compact appearance and about equal size were selected and to these seeds, 4 different salicylic acid (SIGMA, 27301, St. Louis, Missouri, USA) solutions [0 (distilled water), 0.5, 1.0 and $2.0 \mathrm{mM}$ ] has been applied. Applications were applied at room temperature $\left(22^{\circ} \mathrm{C}\right)$ for 24 hours and treated seeds were dried over drying papers. Control group seeds were placed into distilled water at the same temperature for the same duration (Korkmaz et al., 2020). 


\subsection{Boron treatments and germination tests}

Germination tests were conducted in randomized plots design with 4 replications, 50 seeds in each replicate. ISTA (2012) rules were taken into consideration in standard germination tests.

Salicylic acid was applied and seeds that were not applied were subjected to 5 different boron (in the form of boric acid, H3BO3) concentrations (1.0, 2.0, 4.0, 8.0, $\left.16.0 \mathrm{mg} \mathrm{L}^{-1}\right)$, and a control application without boron supplementation into germination medium was also included in experiments. Filter papers to be used seeding, petri dishes and the other glassware were drying sterilized in Pasteur oven. Since the seeds were not contaminated throughout the experiments, they were not sterilized. Germination tests were conducted in petri dishes $(11 \mathrm{~cm}$ in diameter) with filter papers placed inside under climate chamber conditions $\left(25 \pm 1^{0} \mathrm{C}\right.$ temperature, dark and constant relative humidity) (Yıldırım and Güvenç, 2006). Each petri dish was moistened with $8 \mathrm{ml}$ of boron solution at different concentrations and seeds were placed between double-layer of filter papers. Petri dishes were closed, labeled and placed into climate chamber.

Number of germinated seeds was counted 2 days after seed moisturizing and at the same hours of each day. A radicule length of $5 \mathrm{~mm}$ was considered as germinated. During the counts, germinated seeds were removed from the ambient and the remaining seeds were counted. The last count was performed at the end of $14^{\text {th }}$ day and germination percentage (GP, \%) (Li et al., 2007), mean germination time (MGT, day) (Younsheng and Sziklai, 1985), germination index (GI) (AOSA, 1983) and germination energy (GE) (Li et al., 2007) parameters were calculated with the use of the following equations.

$G P=\left(\frac{\text { Germinated seeds }}{\text { Total number of seeds }}\right) \times 100$

$\mathrm{MGT}=\Sigma \operatorname{nixdi} / \mathrm{n}$

where, $n=$ total number of seeds germinated during 14-day experimental period; $n i=$ number of seeds germinated on day di; $d i$ = day during germination period (between 0 and 14).

$\mathrm{GI}=\left(\frac{\text { No. of germinated seeds }}{\text { Day of first count }}\right)+----+\left(\frac{\text { No. of germinated geeds }}{\text { Day of final count }}\right)$

$\mathrm{GE}=\mathrm{b} / \mathrm{c}$

where, $b$ for total number of seeds to germinate, $c$ for germinated seed total in in seven days.

\subsection{Statistical analysis}

The germination percentage, germination energy, germination index and mean germination time data were analyzed using SPSS (version 10.0, SPSS Inc., 1999) software. Analysis of variance was performed on the data, and significant differences among the treatment means were compared by Duncan's multiple range tests $(\mathrm{P}<0.05)$.

\section{Results and Discussion}

Seed germination is regulated by a series of mechanism. A well seed germination contributes to healthy and reliable plant growth and development (Alamri et al., 2018). In present study, pepper, eggplant and watermelon seeds were subjected to different $B\left(0,1.0,2.0,4.0,8.0,16.0 \mathrm{mg} \mathrm{L}^{-1}\right)$ and SA $(0,0.5,1.0$ and $2.0 \mathrm{mM})$ applications and effects of B, SA applications and interactions on germination parameters were determined. Regression analyzes were performed between boron and germination properties, as well as between salicylic acid and germination properties.

\subsection{Germination percentage}

Low B application (1.0 $\left.\mathrm{mg} \mathrm{L}^{-1}\right)$ increased germination percentage of pepper (93.92\%), eggplant (96.60\%) and watermelon (92.94\%) seeds (Table 1). Besides, increasing B doses resulted in toxic effects on seed germination. Decrease in germination ratio started with $2.0 \mathrm{mg} \mathrm{L}^{-1} \mathrm{~B}$ dose and accelerated with increasing boron concentrations and reached to minimum at the greatest $\mathrm{B}$ dose $\left(16.0 \mathrm{mg} \mathrm{L}^{-1}\right)$ (pepper $51.89 \%$, eggplant $50.18 \%$ and watermelon $53.06 \%)$. It was reported in previous studies that low B doses increased germination parameters and high doses negatively influenced germination parameters (Shah et al., 2013; Ashagre, 2014; Alamri et al., 2018). Boron toxicity reduced emergence ratios of maize, tomato and carrot (Banuelos et al., 2009) and increased number of unusual seedlings (Rerkasem and Jamjod, 1997). On the other hand, different SA applications had significant effects on germination percentages (Table 2). The greatest germination percentage in all three species was obtained from 1.0 
mM SA applications (pepper 82.60\%, eggplant 85.24\%, watermelon 81.81\%). Çanakçı (2010) conducted a similar study with barley seeds and reported that SA applications increased the number of germinated seeds. El-Tayeb (2005) indicated that SA applications $(1.0 \mathrm{mM})$ efficiently protected plants against salt-like stress factors in seedling period. According to Güneş et al. (2007), SA applications increased plant growth in stressed or non-stressed ambient. Keeping seeds in SA solution before sowing increased stress tolerance of tomato, bean (Senaratna et al., 2003) and pepper (Korkmaz, 2005) seedlings and improved survival rates of the plants. Dadaşoğlu and Ekinci (2013) indicated that negative effects of salt stress on bean (Phaseolus vulgaris L.) seeds could be prevented with $0.5 \mathrm{mM}$ SA applications.

For germination percentages, $\mathrm{B} \times \mathrm{SA}$ interactions were found to be significant $(\mathrm{P}<0.01)$ (Table 3$)$. The combined application of $1.0 \mathrm{mg} \mathrm{L}^{-1} \mathrm{~B}$ and $0.5 \mathrm{mM} \mathrm{SA}$ increased the germination percentage of all three species to maximum. On the other hand, the lowest germination percentage was obtained from $16.0 \mathrm{mg} \mathrm{L}^{-1} \mathrm{~B}+2.0 \mathrm{mM}$ SA combination. In non-B applied group, the greatest germination percentage was achieved with 0.5 and $1.0 \mathrm{mM} \mathrm{SA}$ in pepper, 0 $\mathrm{mM}$ SA in eggplant and $2.0 \mathrm{mM}$ SA in watermelon. In combined application of $1.0 \mathrm{mg} \mathrm{L}^{-1} \mathrm{~B}$ and SA, differences were observed with species. For instance, while all SA doses had similar effects in pepper, the greatest germination percentage in eggplant and watermelon was obtained from $0.5 \mathrm{mM} \mathrm{SA}$ - applied seeds. In $2.0 \mathrm{mg} \mathrm{L}^{-1} \mathrm{~B}+\mathrm{SA}$ applications, positive effects of additional SA were not observed and non-SA applied seeds had greater applications treatments, the most successful outcomes were achieved with B + 1.0 mM SA combinations. Such a case indicated that $1.0 \mathrm{mM}$ SA applications were successful in alleviating negative impacts of boron stress. On the other hand, the lowest germination percentages in B + SA combinations were obtained from $2.0 \mathrm{mM}$ SA dose.

\subsection{Mean germination time}

Increasing boron concentrations had significant effects on mean germination time of different vegetable seeds (Table 1). In general, increasing mean germination times were observed with increasing $B$ doses. The shortest mean germination time was obtained from $1.0 \mathrm{mg} \mathrm{L}^{-1} \mathrm{~B}$ dose in pepper (3.92 days), eggplant (3.36 days) and watermelon (3.69 days). On the other hand, the longest mean germination time was obtained from $16.0 \mathrm{mg} \mathrm{L}^{-1} \mathrm{~B}$ applications (pepper 4.89 days, eggplant 4.93 days and watermelon 5.09 days). It was reported that high boron levels prolonged mean germination time in watermelon (Farag and Fang, 2014), pepper (Turhan, 2018) and wheat (Kuscu et al., 2018). It was also reported that some applications to seeds (like priming) may increase germination ratio and shorten germination time (Harris and Jones, 1997; Memon et al., 2013; Demirkaya, 2014). It was pointed out that SA, accepted among the hormones effective in stress tolerance (Yıldız et al., 2014), was not essential for germination under normal conditions, but reduce oxidative damage at high stress (salinity) levels and promoted germination (Lee et al., 2010). In this sense, seeds were treated with SA to reduce the effects of B stress and significant correlations were observed between SA and germination time. The $0.5 \mathrm{mM} \mathrm{SA}$ application led to shortest mean germination time (pepper 4.17, eggplant 3.78 and watermelon 4.06 days) and the longest germination time was obtained from $2.0 \mathrm{mM}$ SA applications (pepper 4.32, eggplant 4.05 and watermelon 4.14 days) (Table 2).

For mean germination times, $\mathrm{B} \times \mathrm{SA}$ interactions were found to be significant $(\mathrm{P}<0.01)$. In pepper, eggplant and watermelon seeds, germination time-increasing effect of B doses partially reduced by SA applications (Table 3). Especially in medium and high (4.0, 8.0 and $16.0 \mathrm{mg} \mathrm{L}^{-1}$ ) B applications, $1.0 \mathrm{mM} \mathrm{SA}$ supplementation into growing media significantly shortened germination times. But in the other SA applications, mean germination times were longer. For instance, in medium and high B doses, the greatest number of days to germination in all three vegetables was obtained from non-SA applied seeds. On the other hand, increases were observed in germination times with control (0), 1.0 and $2.0 \mathrm{mg} \mathrm{L}^{-1} \mathrm{~B}$ doses. The longest germination time was obtained from $2.0 \mathrm{mM}$ SA application and the shortest germination time was obtained from non-SA applied seeds.

\subsection{Germination index}

Germination index value increased up to $1.0 \mathrm{mg} \mathrm{L}^{-1} \mathrm{~B}$ dose in pepper (13.59) and watermelon (13.26) and up to $2.0 \mathrm{mg} \mathrm{L}^{-1} \mathrm{~B}$ dose in eggplant (16.71), then further increase in boron concentrations resulted in significant losses in germination index. On the other hand, the lowest germination index was obtained from $16.0 \mathrm{mg} \mathrm{L}^{-1} \mathrm{~B}$ - applied seeds (pepper 10.54, eggplant 10.53 and watermelon 10.51) (Table 1). Similar findings were also reported for peppers (Yermiyahu et al., 2008), tomato and cucumber (Alpaslan and Güneş, 2001). Researchers reported negative effects of high boron concentrations on root and shoot development. In the study, germination index values varied SA applications, the best germination index values were achieved with low SA doses, with $0.5 \mathrm{mM}$ SA in pepper (12.67), $0 \mathrm{mM}$ SA in eggplant (14.88) and $1.0 \mathrm{mM}$ SA in watermelon (13.95). The other SA applications were less effective on germination index (Table 2).

Zahra et al. (2010) reported that SA played an important role in reduction of stress-originated damages. Korkmaz et al. (2017) reported SA doses of between $0.1-1.0 \mathrm{mM}$ as an efficient tool against stress conditions in melon (Cucumis melo L.). In the present study, it was observed also in present study that negative effects of boron toxicity 
could be alleviated and germination parameters of different vegetable seeds could be improved with SA applications. For germination index, $\mathrm{B} \times \mathrm{SA}$ interactions were found to be significant $(\mathrm{P}<0.01)$. The best germination index was achieved with $1.0 \mathrm{mg} \mathrm{L}^{-1} \mathrm{~B}+0.5 \mathrm{mM}$ SA combination in pepper and eggplant and with 1.0 $\mathrm{mg} \mathrm{L}^{-1} \mathrm{~B}+0 \mathrm{mM}$ SA combination in watermelon. The lowest germination index was obtained from $16.0 \mathrm{mg} \mathrm{L}^{-1} \mathrm{~B}+$ 2.0 mM SA combination. In control group without $\mathrm{B}$, the greatest germination index values were obtained from nonSA applications and increasing SA doses negatively influenced germination index. The combined applications of 1.0, $2.0 \mathrm{mg} \mathrm{L}^{-1} \mathrm{~B}$ and different SA doses, control (0 mM SA) and low $(0.5 \mathrm{mM})$ SA doses had more positive effects on germination index. In combined applications of 4.0, 8.0 and $16.0 \mathrm{mg} \mathrm{L}^{-1} \mathrm{~B}$ and different doses of SA, positive effects of $1.0 \mathrm{mM}$ SA were quite distinctive. On the other hand, the lowest germination index values were observed in 4.0 and $16.0 \mathrm{mg} \mathrm{L}^{-1} \mathrm{~B}$ (eggplant and watermelon) $+2.0 \mathrm{mM} \mathrm{SA}$ and $8 \mathrm{mg} \mathrm{L}^{-1} \mathrm{~B}+0.0 \mathrm{mM} \mathrm{SA}$ combinations (Table 3).

Table 1. Effect of boron on the percent seed germination (GP \%), mean germination time (MGT), germination index (GI) and germination energy (GE)

Çizelge 1. Borun tohum çimlenme yüzdesi (\% GP), ortalama çimlenme süresi (MGT), çimlenme indeksi (GI) ve çimlenme enerjisi (GE) üzerine etkisi

\begin{tabular}{|c|c|c|c|c|c|c|c|c|c|c|c|c|}
\hline \multirow{2}{*}{$B\left(\mathrm{mg} \mathrm{L}^{-1}\right)$} & \multicolumn{3}{|c|}{ GP (\%) } & \multicolumn{3}{|c|}{ MGT (Days) } & \multicolumn{3}{|c|}{ GI } & \multicolumn{3}{|c|}{ GE } \\
\hline & $\mathbf{P}$ & EP & WM & $\mathbf{P}$ & EP & WM & $\mathbf{P}$ & EP & WM & $\mathbf{P}$ & EP & WM \\
\hline C & $90.44 \mathrm{~b}$ & $96.52 \mathrm{a}$ & $90.61 \mathrm{~b}$ & $4.07 \mathrm{~d}$ & $3.26 \mathrm{f}$ & $3.71 \mathrm{e}$ & $12.70 \mathrm{c}$ & $16.98 \mathrm{a}$ & $13.15 b$ & $44.59 \mathrm{~b}$ & $66.41 \mathrm{a}$ & $53.54 \mathrm{~b}$ \\
\hline 1.0 & 93.92 a & 96.60 a & 92.94 a & $3.92 \mathrm{e}$ & $3.36 \mathrm{e}$ & $3.69 \mathrm{f}$ & 13.59 a & $16.83 \mathrm{a}$ & $13.26 \mathrm{a}$ & $49.70 \mathrm{a}$ & $60.11 \mathrm{~b}$ & 58.07 a \\
\hline 2.0 & $90.61 \mathrm{~b}$ & $94.80 \mathrm{~b}$ & 89.96 c & $4.13 \mathrm{c}$ & $3.49 \mathrm{~d}$ & $3.77 \mathrm{~d}$ & $12.75 b$ & $16.71 \mathrm{a}$ & $15.04 \mathrm{c}$ & $42.37 \mathrm{c}$ & 57.69 c & $53.95 \mathrm{~b}$ \\
\hline 4.0 & 86.07 c & $81.78 \mathrm{c}$ & $84.44 \mathrm{~d}$ & $4.14 \mathrm{c}$ & $3.99 \mathrm{c}$ & $4.02 \mathrm{c}$ & $12.65 \mathrm{~d}$ & $14.09 \mathrm{~b}$ & $14.16 \mathrm{~d}$ & $39.72 \mathrm{~d}$ & $34.63 \mathrm{~d}$ & $44.22 \mathrm{c}$ \\
\hline 8.0 & $70.08 \mathrm{~d}$ & $71.10 \mathrm{~d}$ & $71.01 \mathrm{e}$ & $4.39 \mathrm{~b}$ & $4.22 \mathrm{~b}$ & $4.31 \mathrm{~b}$ & $11.99 \mathrm{e}$ & $12.83 \mathrm{c}$ & $12.92 \mathrm{e}$ & 25.93 e & $22.21 \mathrm{e}$ & $25.81 \mathrm{~d}$ \\
\hline 16.0 & $51.89 \mathrm{~d}$ & $50.18 \mathrm{e}$ & $53.06 \mathrm{f}$ & $4.89 \mathrm{a}$ & $4.93 \mathrm{a}$ & $5.09 \mathrm{a}$ & $10.54 \mathrm{f}$ & $10.53 \mathrm{~d}$ & $10.51 \mathrm{f}$ & $4.86 \mathrm{f}$ & $8.53 \mathrm{f}$ & $9.61 \mathrm{e}$ \\
\hline
\end{tabular}

As; C “control, 0 mg L-1 B”, B “boron” P "pepper”, EP “eggplant”, WM “watermelon”

Means of the each parameters followed similar letter within the column are not significantly different at the $(\mathrm{P}<0.05)$ level of probability by Duncan's Multiple-Range Test

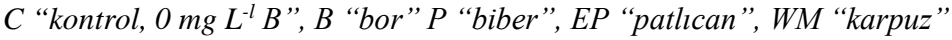

Aynı sütunda yer alan ve aynı harfle başlayan ortalamalar arasındaki farklllı Duncan's Multiple-Range testine göre istatistiksel olarak önemsizdir $(\mathrm{P}<0.05)$.

Table 2. Effect of salicylic acid on the percent seed germination (GP \%), mean germination time (MGT), germination index (GI) and germination energy (GE)

Çizelge 2. Salisilik asidin tohum çimlenme yüzdesi (\% GP), ortalama çimlenme süresi (MGT), çimlenme indeksi (GI) ve çimlenme enerjisi (GE) üzerine etkisi

\begin{tabular}{|c|c|c|c|c|c|c|c|c|c|c|c|c|}
\hline \multirow{2}{*}{$\mathrm{SA}(\mathrm{mM})$} & \multicolumn{3}{|c|}{ GP (\%) } & \multicolumn{3}{|c|}{ MGT (Days) } & \multicolumn{3}{|c|}{ GI } & \multicolumn{3}{|c|}{ GE } \\
\hline & $\mathbf{P}$ & EP & WM & $\mathbf{P}$ & EP & WM & $\mathbf{P}$ & EP & WM & $\mathbf{P}$ & EP & WM \\
\hline $\mathbf{0}$ & $79.35 c$ & $81.80 \mathrm{c}$ & $80.13 \mathrm{~b}$ & $4.31 \mathrm{~b}$ & $3.87 \mathrm{~b}$ & $4.09 \mathrm{~b}$ & $12.32 \mathrm{c}$ & $14.88 \mathrm{a}$ & $13.91 \mathrm{~b}$ & $34.22 \mathrm{~b}$ & $44.36 \mathrm{a}$ & $40.48 \mathrm{~b}$ \\
\hline 0.5 & $80.90 \mathrm{~b}$ & $82.52 \mathrm{~b}$ & $80.51 \mathrm{~b}$ & $4.17 \mathrm{~d}$ & $3.78 \mathrm{~d}$ & $4.09 \mathrm{~b}$ & $12.67 \mathrm{a}$ & $14.81 \mathrm{a}$ & $13.83 \mathrm{c}$ & $40.01 \mathrm{a}$ & $42.16 \mathrm{c}$ & $43.71 \mathrm{a}$ \\
\hline 1.0 & 82.66 a & $85.24 \mathrm{a}$ & 81.81 a & $4.22 \mathrm{c}$ & $3.80 \mathrm{c}$ & $4.14 \mathrm{a}$ & $12.36 \mathrm{~b}$ & $14.85 \mathrm{a}$ & $13.95 \mathrm{a}$ & 33.52 c & $43.75 \mathrm{~b}$ & $40.75 \mathrm{~b}$ \\
\hline 2.0 & $79.09 \mathrm{c}$ & $77.75 \mathrm{~d}$ & 78.89 c & $4.32 \mathrm{a}$ & $4.05 \mathrm{a}$ & $4.07 \mathrm{C}$ & $12.12 \mathrm{~d}$ & $14.11 \mathrm{~b}$ & $13.67 \mathrm{~d}$ & $30.36 \mathrm{~d}$ & $36.11 \mathrm{~d}$ & $40.53 \mathrm{~b}$ \\
\hline
\end{tabular}

As; SA “salicylic acid” P "pepper”, EP “eggplant”, WM “watermelon”

Means of the each parameters followed similar letter within the column are not significantly different at the $(\mathrm{P}<0.05)$ level of probability by Duncan's Multiple-Range Test

SA “salisilik asit”, P "biber”, EP "patlican", WM "karpuz"

Aynı sütunda yer alan ve aynı harfle başlayan ortalamalar arasındaki farklllık Duncan's Multiple-Range testine göre istatistiksel olarak önemsizdir $(\mathrm{P}<0.05)$. 
Table 3. Boron $\times$ salicylic acid interactions for the percent seed germination (GP \%), mean germination time (MGT), germination index (GI) and germination energy (GE)

Çizelge 3. Tohum çimlenme yüzdesi (\% GP), ortalama çimlenme süresi (MGT), çimlenme indeksi (GI) ve çimlenme enerjisi (GE) üzerine Bor $\times$ salisilik asit interaksiyonları

\begin{tabular}{|c|c|c|c|c|c|c|c|c|c|c|c|c|c|}
\hline \multirow{2}{*}{$\begin{array}{c}\text { B } \\
\left(\mathrm{mg} \mathrm{L}^{-1}\right)\end{array}$} & \multirow{2}{*}{$\begin{array}{c}\text { SA } \\
(\mathrm{mM})\end{array}$} & \multicolumn{3}{|c|}{$G(\%)$} & \multicolumn{3}{|c|}{ MGT (Days) } & \multicolumn{3}{|c|}{ GPI } & \multicolumn{3}{|c|}{ GE } \\
\hline & & $\mathbf{P}$ & EP & WM & $\mathbf{P}$ & EP & WM & $\mathbf{P}$ & EP & WM & $\mathbf{P}$ & EP & WM \\
\hline \multirow{4}{*}{ C } & 0 & $90.06 \mathrm{c}$ & $97.00 \mathrm{ac}$ & $89.56 \mathrm{df}$ & $3.76 p$ & $3.10 \mathrm{v}$ & $3.64 \mathrm{o}$ & $13.64 \mathrm{c}$ & $17.33 \mathrm{ab}$ & $15.48 \mathrm{~b}$ & $56.75 \mathrm{~b}$ & $74.15 \mathrm{a}$ & $52.00 \mathrm{f}$ \\
\hline & 0.5 & $90.94 \mathrm{bc}$ & $96.88 \mathrm{bc}$ & $90.81 \mathrm{~cd}$ & $4.06 n$ & $3.22 \mathrm{t}$ & $3.68 n$ & $12.74 \mathrm{~g}$ & $16.34 \mathrm{c}$ & $15.20 \mathrm{~d}$ & 46.80 de & $66.00 \mathrm{c}$ & $56.11 \mathrm{~d}$ \\
\hline & 1.0 & 90.75 bc & $96.38 \mathrm{~cd}$ & 90.00 de & 4.201 & $3.24 t$ & 3.781 & $12.23 \mathrm{o}$ & $17.44 \mathrm{ab}$ & 14.861 & $32.48 \mathrm{k}$ & $65.95 \mathrm{c}$ & $52.00 \mathrm{f}$ \\
\hline & 2.0 & $90.00 \mathrm{c}$ & $95.81 \mathrm{~d}$ & 92.06 bc & $4.28 \mathrm{~g}$ & $3.48 p$ & $3.74 \mathrm{~m}$ & $12.20 \mathrm{p}$ & $16.26 \mathrm{c}$ & $15.08 \mathrm{gh}$ & $42.33 \mathrm{~g}$ & $59.50 \mathrm{f}$ & $54.05 \mathrm{e}$ \\
\hline \multirow{4}{*}{1.0} & 0 & $94.04 \mathrm{a}$ & $97.56 \mathrm{ab}$ & $93.75 \mathrm{a}$ & $3.70 \mathrm{r}$ & $3.13 \mathrm{u}$ & $3.62 \mathrm{p}$ & $14.44 \mathrm{~b}$ & $17.54 \mathrm{ab}$ & $15.62 \mathrm{a}$ & $54.61 \mathrm{c}$ & $64.20 \mathrm{~d}$ & $59.95 \mathrm{~b}$ \\
\hline & 0.5 & 94.13 a & $97.90 \mathrm{a}$ & 93.81 a & $3.59 \mathrm{~s}$ & $3.23 \mathrm{t}$ & $3.68 n$ & $14.65 \mathrm{a}$ & $17.86 \mathrm{a}$ & $15.16 \mathrm{e}$ & $65.76 \mathrm{a}$ & $62.00 \mathrm{e}$ & $62.08 \mathrm{a}$ \\
\hline & 1.0 & 94.27 a & $96.50 \mathrm{~cd}$ & $92.50 \mathrm{ab}$ & $4.17 \mathrm{jk}$ & $3.51 \mathrm{p}$ & $3.74 \mathrm{~m}$ & $12.71 \mathrm{~h}$ & $16.25 c$ & $15.11 \mathrm{fg}$ & 38.161 & $62.13 \mathrm{e}$ & $54.25 \mathrm{e}$ \\
\hline & 2.0 & $93.25 \mathrm{a}$ & $94.44 \mathrm{e}$ & 91.69 bc & 4.191 & $3.56 \mathrm{o}$ & $3.74 \mathrm{~m}$ & $12.55 \mathrm{j}$ & $16.22 \mathrm{c}$ & $15.14 \mathrm{e}$ & $40.25 \mathrm{~h}$ & 52.131 & $56.00 \mathrm{~d}$ \\
\hline \multirow{4}{*}{2.0} & 0 & $91.94 \mathrm{~b}$ & $96.06 \mathrm{~cd}$ & 91.93 bc & $4.05 n$ & $3.30 \mathrm{~s}$ & 3.781 & $13.33 \mathrm{~d}$ & $17.37 \mathrm{ab}$ & $15.11 \mathrm{fg}$ & $39.75 \mathrm{~h}_{1}$ & $68.00 \mathrm{~b}$ & $51.95 \mathrm{f}$ \\
\hline & 0.5 & $89.93 \mathrm{c}$ & $96.00 \mathrm{~cd}$ & 90.06 de & $4.09 \mathrm{~m}$ & $3.33 r$ & $3.60 \mathrm{p}$ & $12.69 \mathrm{~h}$ & $17.13 \mathrm{~b}$ & 15.25 c & $47.93 \mathrm{~d}$ & $58.00 \mathrm{~g}$ & $58.18 \mathrm{c}$ \\
\hline & 1.0 & $90.31 \mathrm{c}$ & 96.81 bd & $89.25 \mathrm{df}$ & 4.131 & $3.59 n$ & $3.89 \mathrm{k}$ & $12.64_{1}$ & $16.34 \mathrm{c}$ & $14.73 \mathrm{j}$ & $45.91 \mathrm{e}$ & $53.75 \mathrm{~h}$ & $49.92 \mathrm{~g}$ \\
\hline & 2.0 & $90.25 \mathrm{c}$ & $90.31 \mathrm{f}$ & $88.58 \mathrm{f}$ & $4.24 \mathrm{~h}$ & $3.71 \mathrm{~m}$ & 3.801 & $12.36 \mathrm{~m}$ & $16.01 \mathrm{c}$ & $15.06 \mathrm{~h}$ & $35.90 \mathrm{j}$ & $51.00 \mathrm{j}$ & $55.75 \mathrm{~d}$ \\
\hline \multirow{4}{*}{4.0} & 0 & $86.65 \mathrm{~d}$ & 80.061 & $85.06 \mathrm{~g}$ & $4.27 \mathrm{~g}$ & $4.11 \mathrm{~h}$ & $4.06 \mathrm{~h}$ & $12.28 \mathrm{n}$ & $14.12 \mathrm{de}$ & 14.341 & $40.32 \mathrm{~h}$ & $32.00 \mathrm{~m}$ & $46.06 \mathrm{~h}$ \\
\hline & 0.5 & $86.63 \mathrm{~d}$ & $82.75 \mathrm{~h}$ & $85.75 \mathrm{~g}$ & $4.06 n$ & $3.92 \mathrm{k}$ & 4.011 & $12.86 \mathrm{f}$ & 14.19 de & 13.831 & $44.06 \mathrm{f}$ & 37.981 & $46.90 \mathrm{~h}$ \\
\hline & 1.0 & $86.31 \mathrm{~d}$ & $86.68 \mathrm{~g}$ & $85.19 \mathrm{~g}$ & $4.01 \mathrm{o}$ & 3.871 & $3.97 \mathrm{j}$ & $12.97 \mathrm{e}$ & $14.39 \mathrm{~d}$ & $14.65 \mathrm{k}$ & $36.17 \mathrm{j}$ & $42.00 \mathrm{k}$ & $43.90_{1}$ \\
\hline & 2.0 & $84.69 \mathrm{e}$ & $77.63 \mathrm{j}$ & $81.75 \mathrm{~h}$ & $4.22 \mathrm{~h}_{1}$ & 4.071 & $4.05 \mathrm{~h}$ & 12.491 & $13.68 \mathrm{ef}$ & 13.811 & $38.34_{1}$ & $26.55 n$ & $40.00 \mathrm{j}$ \\
\hline \multirow{4}{*}{8.0} & 0 & $65.64 \mathrm{~h}$ & 70.251 & $70.25 \mathrm{j}$ & $4.92 \mathrm{~b}$ & $4.51 \mathrm{e}$ & $4.45 \mathrm{~d}$ & $10.65 \mathrm{t}$ & $12.01 \mathrm{~h}$ & $12.65 \mathrm{r}$ & $11.67 \mathrm{~m}$ & $19.85 \mathrm{p}$ & $23.95 \mathrm{~m}$ \\
\hline & 0.5 & $71.94 \mathrm{~g}$ & $71.55 \mathrm{k}$ & $70.38 \mathrm{j}$ & $4.18 \mathrm{ij}$ & $4.20 \mathrm{f}$ & $4.29 \mathrm{f}$ & $12.51 \mathrm{kl}$ & $12.92 \mathrm{~g}$ & $12.97 \mathrm{o}$ & $31.39 \mathrm{k}$ & $21.00 \mathrm{o}$ & 29.771 \\
\hline & 1.0 & $76.98 \mathrm{f}$ & $76.88 \mathrm{j}$ & $75.44_{1}$ & $4.15 \mathrm{kl}$ & $3.99 \mathrm{j}$ & $4.15 \mathrm{~g}$ & $12.52 \mathrm{k}$ & $13.39 \mathrm{fg}$ & $13.30 \mathrm{n}$ & $36.37 \mathrm{j}$ & $26.50 n$ & $32.18 \mathrm{k}$ \\
\hline & 2.0 & $65.76 \mathrm{~h}$ & $65.75 \mathrm{~m}$ & $68.00 \mathrm{k}$ & $4.31 \mathrm{f}$ & $4.17 \mathrm{~g}$ & $4.35 \mathrm{e}$ & $12.19 \mathrm{p}$ & $13.01 \mathrm{~g}$ & $12.76 \mathrm{p}$ & 24.301 & $21.50 \mathrm{o}$ & 29.351 \\
\hline \multirow{4}{*}{16.0} & 0 & $48.13 j$ & 49.91 o & $50.199 \mathrm{n}$ & $5.30 \mathrm{a}$ & $5.07 \mathrm{~b}$ & $5.18 \mathrm{a}$ & $9.58 \mathrm{v}$ & $10.42 \mathrm{k}$ & $10.27 \mathrm{u}$ & $2.23 \mathrm{o}$ & $7.93 \mathrm{~s}$ & $8.10 \mathrm{p}$ \\
\hline & 0.5 & 51.811 & $50.05 \mathrm{o}$ & $52.31 \mathrm{~m}$ & $4.89 \mathrm{c}$ & $4.76 \mathrm{c}$ & $5.11 \mathrm{~b}$ & $10.52 \mathrm{u}$ & $10.93 \mathrm{ij}$ & $10.5 \mathrm{t}$ & $4.16 n$ & $8.00 \mathrm{~s}$ & $10.05 \mathrm{o}$ \\
\hline & 1.0 & $57.00 \mathrm{~h}$ & $58.18 n$ & 58.501 & $4.65 \mathrm{e}$ & $4.60 \mathrm{~d}$ & $4.89 \mathrm{c}$ & $11.12 \mathrm{r}$ & 11.311 & $11.06 \mathrm{~s}$ & $12.02 \mathrm{~m}$ & $12.18 \mathrm{r}$ & $12.25 n$ \\
\hline & 2.0 & 50.631 & $42.56 \mathrm{p}$ & $51.25 \mathrm{mn}$ & $4.72 \mathrm{~d}$ & $5.30 \mathrm{a}$ & 5.17 a & $10.95 \mathrm{~s}$ & 9.471 & $10.16 \mathrm{v}$ & $1.05 \mathrm{o}$ & $6.00 \mathrm{t}$ & $8.03 \mathrm{p}$ \\
\hline
\end{tabular}

As; C “control, 0 mg L ${ }^{-1}$ B”, B “boron”, SA “salicylic acid” P “pepper”, EP “eggplant”, WM “watermelon”

Means of the each parameters followed similar letter within the column are not significantly different at the $(\mathrm{P}<0.05)$ level of probability by Duncan's Multiple-Range Test

$C$ “kontrol, 0 mg L ${ }^{-1}$ B”, B “bor” SA "salisilik asit” $P$ “biber”, EP “patlican”, WM "karpuz”

Aynı sütunda yer alan ve aynı harfle başlayan ortalamalar arasındaki farklllk Duncan's Multiple-Range testine göre istatistiksel olarak önemsizdir $(\mathrm{P}<0.05)$.

\subsection{Germination energy}

Germination energy values significantly varied with the boron doses (Table 1). The greatest germination energy was obtained from $1.0 \mathrm{mg} \mathrm{L}^{-1} \mathrm{~B}$ dose in pepper (49.70) and watermelon (58.07) and from the control application without B in eggplant (66.41). As it was indicated by Kuşçu et al. (2018), increasing boron concentrations of germination media significantly reduced germination energy values. The lowest values were obtained from $16.0 \mathrm{mg}$ $\mathrm{L}^{-1} \mathrm{~B}$ applications (pepper 4.86, watermelon 8.53 and eggplant 9.61). Similarly, the greatest germination energy values were obtained from $0.5 \mathrm{mM}$ SA applications in pepper (40.1) and watermelon (43.71) and from $0 \mathrm{mM} \mathrm{SA}$ applications in eggplant (44.36). Increasing SA doses negatively influenced germination energy values and the lowest values were observed in $2.0 \mathrm{mM}$ SA applications (Table 2).

As can be inferred from Table 3, interactions of $\mathrm{B} \times \mathrm{SA}$ had significant effects $(\mathrm{P}<0.01)$ on germination energy values. The greatest germination energy was obtained from $1.0 \mathrm{mg} \mathrm{L}^{-1} \mathrm{~B} \times 0.5 \mathrm{mM}$ SA combination in pepper and watermelon and from the control treatment without B and SA in eggplant. Sakhabutdinova et al. (2003) pointed out the promoting effect of SA treatments on germination parameters; in contrast Rajasekaran et al. (2002) indicated insignificant effects of SA on germination. In present study, SA treatments yielded positive outcomes through increasing germination energy of the seeds subjected to boron toxicity. Such positive effects could clearly be seen at high B concentrations ( 8.0 and $16.0 \mathrm{mg} \mathrm{L}^{-1}$ ). At both $\mathrm{B}$ concentrations, the greatest germination energy values were obtained from 1.0 mM SA- applied seeds. But, positive effects reduced in 8.0 and $16.0 \mathrm{mg} \mathrm{L}^{-1} \mathrm{~B} \times 0.0$ and $2.0 \mathrm{mM}$ SA combinations. Similar with these findings, Culpan and Arslan (2018) indicated negative effects of high SA and $\mathrm{GA}_{3}$ doses on plant growth. Korkmaz et al. (2020) reported that SA slightly improved plant (canola, Brassica napus 
L.) growth and development under salt stress. In the other combinations, germination energy values varied with the species. For instance, in pepper and watermelon, 1.0, 2.0 and $4.0 \mathrm{mg} \mathrm{L}^{-1} \mathrm{~B}+0.5 \mathrm{mM}$ SA applications positively influenced germination energy of the seeds. On the other hand, in eggplant, control, 1.0 and $2.0 \mathrm{~B} \mathrm{mg} \mathrm{L}^{-1} \mathrm{~B}+0 \mathrm{mM}$ SA applications yielded better outcomes. In 1.0, 2.0 and 4.0 B mg L-1 and SA combinations, 1.0 and mostly $2.0 \mathrm{mM}$ SA doses reduced to germination energy to the lowest levels.

\subsection{Relationships between the rates of boron and salicylic acid and germination properties}

The relationships between B levels and germination characteristics are given in Fig. 1. Significant 2nd order polynomial relationships were obtained between the germination percentage of pepper, eggplant and watermelon seeds and $B$ levels. The determination coefficients $\left(R^{2}=0.97\right.$ for pepper, $R^{2}=0.99$ for eggplant, and $R^{2}=0.98$ for watermelon) of these relationships are quite high $(\mathrm{P}<0.01)$. This result shows that $97 \%$ to $99 \%$ of the total variation in the germination percentage of 3 different plant seeds can be explained by these equations. Significant linear relationships were obtained between different $\mathrm{B}$ doses and mean germination times of 3 different plant seeds, and germination times of seeds were prolonged with increasing B doses. A linear relationship was found between the germination index of pepper and eggplant seeds and B levels, and a curvilinear (2nd order polynomial) relationship was obtained between the germination index of watermelon seeds and the B levels. In addition, a linear relationship was obtained between the germination energy of pepper and watermelon seeds and B levels, and a curvilinear (2nd order polynomial) relationship was found for eggplant seeds.

The relationships between salicylic acid levels under different B doses and the germination characteristics of 3 different plant seeds are shown in Fig. 2. The relationships between salicylic acid and germination properties mentioned above are defined by quadratic functions. However, in general, relationships between salicylic acid and germination properties were found to be weaker (lower coefficients of determination) than those between B and germination properties.


Figure 1. Some relationships between the B levels and the germination properties [germination percentage (GP), mean germination time (MGT), germination index (GI), and germination energy (GE)] $* \mathrm{P}<0.05, * * \mathrm{P}<0.01$

Şekil 1. B seviyeleri ile çimlenme özellikleri [çimlenme yüzdesi (GP), ortalama çimlenme süresi (MGT), çimlenme indeksi (GI), çimlenme enerjisi (GE)] arasındaki bazı ilişskiler $* P<0.05, * * P<0.01$ 

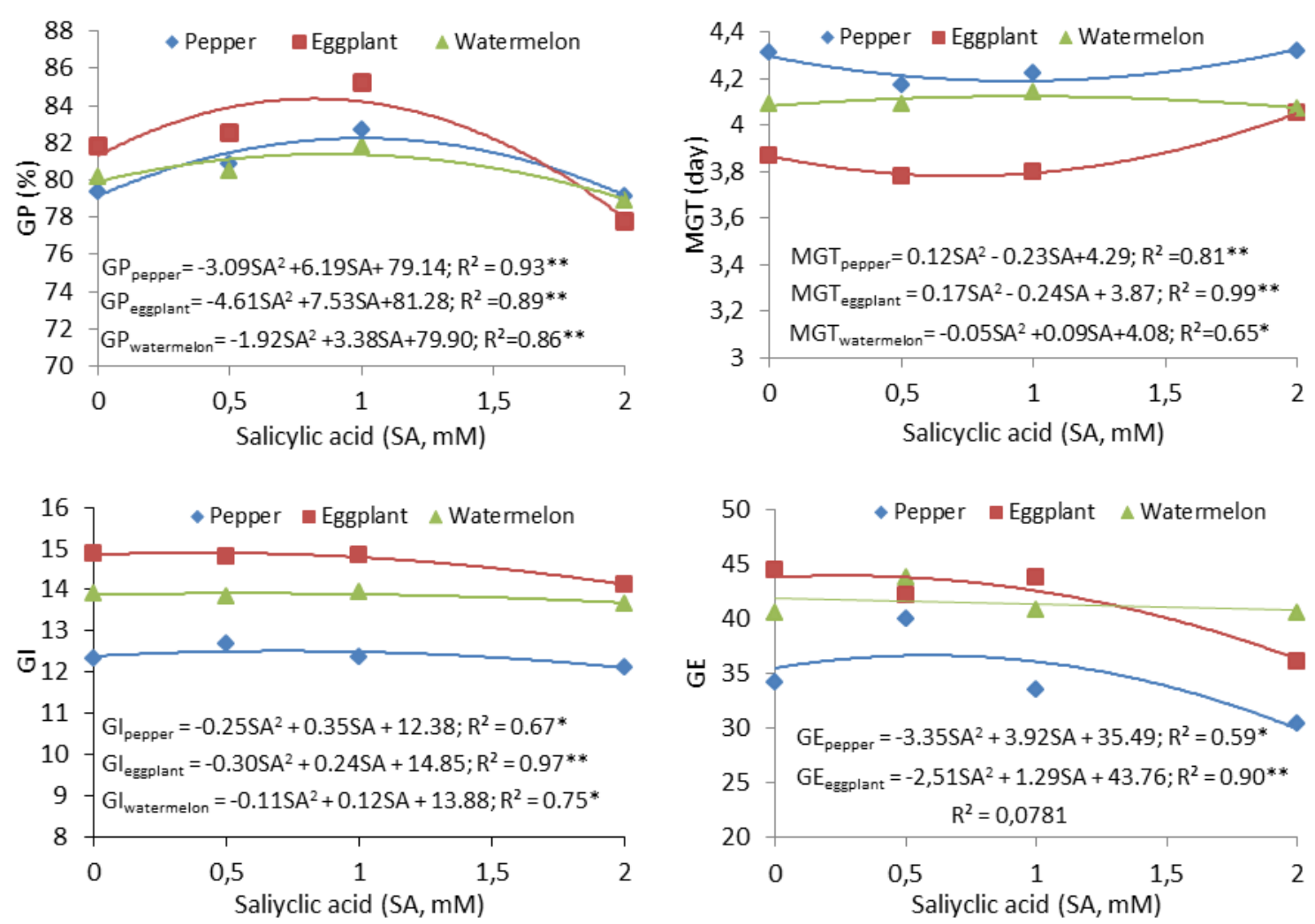

Figure 2. Some relationships between the salicylic acid (SA) levels and the germination properties [germination percentage (GP), mean germination time (MGT), germination index (GI), and germination energy (GE)] ${ }^{*} \mathrm{P}<0.05,{ }^{*} \mathrm{P}<0.01$

Şekil 2. Farklı salisilik asit (SA) seviyeleri ile çimlenme özellikleri [çimlenme yüzdesi (GP), ortalama çimlenme süresi (MGT), çimlenme indeksi (GI), çimlenme enerjisi (GE)] arasındaki bazı ilişkiler ${ }^{*} P<0.05, * * P<0.01$

\section{Conclusion}

Present findings revealed that B toxicity may generate serious problems. On the other hand, our findings show that the harmful effects of boron can be reduced with SA application. The greatest germination percentage, germination index and germination energy values were obtained from $1.0 \mathrm{mg} \mathrm{L}^{-1} \mathrm{~B}$ - applied seeds of three different vegetable species (pepper, eggplant and watermelon). However, increasing boron concentrations prolonged the mean germination time and negatively influenced germination parameters. Especially under medium and high (4.0 and $8.0,16.0 \mathrm{mg} \mathrm{L}^{-1}$ ) B stress, $1.0 \mathrm{mM} \mathrm{SA}$ applications increased germination parameters and reduced the negative effects of boron on germination parameters. The SA doses of over $2.0 \mathrm{mM}$ had negative effects on investigated parameters. The 0 and $0.5 \mathrm{mM}$ SA doses had quite limited effects mostly on non-B applied or slightly on low B (0 and $1.0 \mathrm{mg} \mathrm{L}^{-1}$ ) treated seeds. For practical use of SA, further research is recommended to be conducted with greater number of culture crops and to investigate the effects in different growth stages of the plants.

\section{References}

Adriano, D.C., 1986. Trace Elements in the Terrestrial Environment, Springer Verlag, New York, pp. 73-79.

Alamri, S.A., Siddiqui, M.H., Al-Khaishani, M.Y., Hayssam, M.A., 2018. Boron induces seed germination and seedling growth of Hordeum vulgare L. under $\mathrm{NaCl}$ stress. Journal of Advances in Agriculture, 8(1): 12241234. ISSN: 2349-0837.

Alpaslan, M. and Güneş, A., 2001. Interactive effects of boron and salinity stress on the growth, membrane permeability, and mineral composition of tomato and cucumber plants. Plant and Soil, 236: 123-128.

AOSA, 1983. Association of Official Seed Analysis. In Seed vigor testing handbook.

Ashagre, H., Hamza, I.A., Fita, U. and Nedesa, W., 2014. Influence of boron on seed germination and seedling growth of wheat (Triticum aestivum L.). African Journal of Plant Science, 8(2): 133-139. doi: 10.5897/AJPS2014.1148. 
Baktır İ., 2010. Bitki büyüme düzenleyicileri özellikleri ve tarımda kullanımları. Hasad Yayıncılık, 112p (in Turkish).

Bañuelos, G.S., Robinson, J., Da Roche, J., 2009. Developing selenium-enriched animal feed and biofuel from canola planted for managing Se-laden drainage waters in the Westside of Central California. Int. J. Phytoremediation, 12: 243-254. doi: 10.1080\%2F15226510903563850.

Baran, I., Kulaz, H., Curka, M., Tunçtürk, M., Oral, H., 2019. The impact of salt ( $\mathrm{NaCl})$ stress on germination characteristics of salicylic acid (SA) pretreated black chickpeas (Cicer arietinum L.) seed. ISPEC International Conference on Agriculture, Animal Science and Rural Development-II, (1): 55-57, Van, Turkey.

Boukraa, D., Belabid, L., Benabdelli, K., Bennabi, F., 2015. Implication of salicylic acid in chickpea growth to salt resistance. Advances in Environmental Biology, 9(27): 270-277. ISSN: 1995-0756.

Brdar-Jokanovic, M., 2020. Boron toxicity and deficiency in agricultural plants. International Journal of Molecular Sciences, 21: 1424. doi:10.3390/ijms21041424.

Çanakçı, S., 2010. Barley (Hordeum vulgare L. cv.) effects of salicylic acid and ferulic acid on germination of barley (Hordeum vulgare L. cv.) seeds, several growth parameters and pigment amounts. Frrat Univ. Journal of Science, 22 (1): 37-45.

Culpan, E. and Arslan, B., 2018. Effects of applications salicylic acid on seed yield and some quality traits of safflower cultivars (Carthamus tinctorius L.). Academic Journal of Agr., 7(2):173-178. doi:10.29278/azd.476336.

Dadaşoglu, E. and Ekinci, M., 2013. Effects of different degrees of temperature, salt and salicylic acid applications on seed germination of bean (Phaseolus vulgaris L.). Atatürk Univ., J. of the Agricultural Faculty, 44 (2): 145150. ISSN: 1300-9036.

Datta, K.S., Varma, S.K., Angrish, R., Kumar, B., Kumari, P., 1997. Alleviation of salt stress by plant growth regulators in Triticum aestivum L. Biologia Plantarum, 40 (2): 269-275.

Demirkaya, M., 2014. Improvement in tolerance to salt stress during tomato cultivation. Turk J. Biol., 38: 193-199. doi:10.3906/biy-1307-6.2 doi:10.4067\%2FS0718-95162017005000032.

El-Tayeb, M.A., 2005. Response of barley grains to the interactive effect of salinity and salicylic acid. Plant Growth Regulation, 45: 215-224. doi: 10.1007/s10725-005-4928-1.

Farag, M. and Fang, Z.M., 2014. Effect of boron toxicity stress on seed germination, root elongation and early seedling development of watermelon Citrullus lanatus Thumb. J. Anim. Plant Sci., 21: 3313-3325. ISSN: 20717024.

Güneş, A., Pilbeam, D.J., İnal, A., Bağc1, E.G. and Cobani, S., 2007. Influence of silicon on antioxidant mechanisms and lipid peroxidation in chickpea (Cicer arietinum L.) cultivars under drought stress. Journal of Plant Interactions, 2(2): 105-113. doi:10.1080/17429140701529399.

Harris, S. and Jones, D.B., 1997. Optimization of the polymerase chain reaction. British Journal of Biomedical Science, 54: 166-173.

Hayat, Q., Hayat, S., Irfan, M. and Ahmad, A., 2010. Effect of exogenous salicylic acid under changing environment: A review. Environmental and Experimental Botany, 68: 14-25. doi:10.1016/j.envexpbot.2009.08.005.

ISTA, 2012. International Rules for Seed Testing. Edition 2012. International Seed Testing Association, Bassersdorf, Switzerland.

Kaydan, D. and Yağmur, M., 2006. Effects of different salicylic acid doses and treatments on wheat (Triticum aestivum L.) and Lentil (Lens culinaris Medik.) yield and yield components. Journal of Agricultural Sciences, 12 (3): 285-293. ISSN: 1300-7580.

Korkmaz, A., Özbay, N., Tiryaki, I., Nas, M.N., 2005. Combining priming and plant growth regulators improves muskmelon germination and emergence at low temperatures. European Journal of Horticultural Science, 70(1): 29-34. ISSN: 1611-4426.

Korkmaz, A., Uzunlu, M., Demirkıran, A.R., 2017.Treatment with acetyl salicylic acid protects muskmelon seedlings against drought stress. Acta Physiol. Plant, 29: 503-508. doi: 10.1007/s11738-007-0060-3.

Korkmaz, K., Akgün, M., Kırlı, A., Özcan, M.M., Dede, Ö., Kara, Ş.M., 2020. Effects of gibberellic acid and salicylic acid applications on some physical and chemical properties of rapeseed (Brassica napus L.) grown under salt stress. Turkish Journal of Agriculture - Food Science and Technology, 8(4): 873-881. doi:10.24925/turjaf.v8i4.873-881.3044.

Kumlay, A.M. and Eryiğit, T., 2011. Growth and development regulators in plants: plant hormones. Iğdır Univ. J. Inst. Sci. \& Tech., 1 (2): 47-56.

Kuşçu, H., Kurtulmuş, E., Arslan, B., Karakuş, E., Kumraltekin, E., Uçan, İ.E., Aşıı, M.C., 2018. Effects of different boron concentrations on germination of bread wheat cultivars, Süleyman Demirel University Journal of the Faculty of Agriculture $1^{\text {st }}$ International Agricultural Structures and Irrigation Congress, pp. 319-327. 
Lee, S., Kim, S.G. and Park, C.M., 2010. Salicylic acid promotes seed germination under high salinity by modulating antioxidant activity in Arabidopsis. New Phytologist, 188: 626-637. doi: 10.1111/j.14698137.2010.03378.x.

Li, C., Feng, S., Shao, Y., Jiang, L., Lu, X., Hao, X., 2007. Effects of arsenic on seed germination and physiological activities of wheat seedlings. Journal of Environmental Sciences, 19: 725-732. doi: 10.1016/S10010742(07)60121-1.

Memon, N.N., Gandahi, M.B., Pahoja, V.M. and Sarif, N., 2013. Response of seed priming with germination and seedling sprouts of broccoli. International Journal of Agricultural Science and Research, 3(2): 183-194.

Rajasekaran, L.R., Stiels, A. and Caldwell, C.D., 2002. Stand establishment in processing carrots: Effects of various temperature regimes on germination and the role of salicylic acid in promoting germination at low temperatures. Canadian J. of Sci., 82: 443-500.

Rajjou, L., Belghazi, M., Huguet, R., Robin, C., Moreau, A., Job, C., Job, D., 2006. Proteomic investigation of the effect of salicylic acid on Arabidopsis seed germination and Establishment of early defense mechanism, Plant Physiol., 141: 910-923. doi:10.1104\%2Fpp.106.082057.

Rerkasem, B. and Jamjod, S., 1997. Boron deficiency induced male sterility in wheat (Triticum aestivum L.) and implification for plant breeding. Euphytica (In Press Ref.MS No EUPH 4247), 140 p.

Sakhabutdinova, A.R., Fatkhutdinova, D.R., Bezrukova, M.V. and Shakirova, F.M., 2003. Salicylic acid prevents the damaging action of stress factors on wheat plants. Bulg. J. Plant Physiol., special issue, 314-319.

Sarafi, E., Siomos, A., Tsouvaltzis, P., Tsouvaltzis, C., Therios, I., 2017. Boron toxicity effects on grafted and nongrafted pepper (Capsicum annuum) plants. Journal of Soil Science and Plant Nutrition, 17 (2): 441-460.

Senaratna, T., Merritt, D., Dixon, K., Bunn, E., Touchell, D., Sivasithamparam, K., 2003. Benzoic acid may act as the functional group in salicylic acid and derivatives in the induction of multiple stress tolerance in plants. Plant Growth Regulation, 39: 77-81.

Shah, M.H.R., Bokhari, T.Z., Younis, U., 2013. Boron irrigation effect on germination and morphological attributes of Zea mays cultivars (Cv.Afghoee \& Cv.Composite). Int. J. Sci. Engi. Res., 4(8):1563-1569. ISSN: 2229-5518.

Singh, P., Chaturvedi, V., Kumar, B., 2010. Effects of salicylic acid on seedling growth and nitrogen metabolism in cucumber (Cucumis sativus L.). Journal of Stress Physiology and Biochemistry, 6(3): 103-113. ISSN: 1997-0838

Turhan, A., 2018. Effects of different boron applications on red pepper germination and seedling development. International Agriculture, Environment and Health Congress pp. 265-271, 26-28 October, Aydın, Turkey.

Ünver, M.C. and Tilki, F., 2012. Salinity, germination promoting chemicals, temperature and light effects on seed germination of Anethum graveolens L. Bulgarian J. of Agri. Sci., 18 (6): 1005-1011.

Yermiyahu, U., Ben-Gal, A., Keren, R. and Reid, R.J., 2008. Combined effect of salinity and excess boron on plant growth and yield. Plant \& Soil, 304: 73-87. doi:10.1007\%2Fs11104-007-9522-z.

Yıldırım, E. and Güvenç, I., 2006. Salt Tolerance of Pepper Cultivars during Germination and Seedling Growth. Turk. J. Agric. For., 30: 347-353.

Yıldız, M., Terzi, H., Akçalı, N., 2014. Salicylic acid and polyamines in plant salt stress tolerance. AKU J. Sci. Eng. 14: 7-22. doi:10.5578\%2Ffmbd.7763.

Younsheng, C. and Sziklai, O., 1985. Preliminary study on the germination of Toora sinensis (A.JUSS). Roem. seed from eleven Chinese provenances. For. Ecol. Manage., 10: 269-281.

Zahra, S., Amin, B., Mehdi, M., 2010.The salicylic acid effect on the tomato (Lycopersicum esculentum Mill.) germination, growth and photosynthetic pigment under salinity stress $(\mathrm{NaCl})$. Journal of Stress Physiology \& Biochemistry, 6(3):4-16. ISSN: 1997-0838. 